http://www.inass.org/

\title{
Applying Wireless Sensor Networks in an Online Monitoring and Energy Management System for Industrial Motors
}

\author{
Jingtao Hu \\ ${ }^{1}$ Key Laboratory of Industrial Informatics, Shenyang Institute of Automation, Chinese Academy of Sciences, \\ Shenyang 110016, China \\ ${ }^{2}$ Graduate School of the Chinese Academy of Sciences, Beijing 100039, China \\ * Corresponding author's Email: hujingtao@sia.cn
}

\begin{abstract}
This paper presents an application of wireless sensor networks (WSN) to an in-service motor monitoring and energy management system. Based on the IEEE 802.15.4 standard, wireless sensor devices are developed in the proposed system. The motor current and voltage signals are acquired and analyzed by a DSP device to get the condition of motors, and the results are transmitted over the wireless network to a central supervisory station (CSS), where they are stored, displayed and analyzed to meet the requirement of motor monitoring and energy management. This approach greatly reduces the transmission time. That makes the proposed system acceptable in real-time cases. The wireless sensor devices are demonstrated and the test results are given.
\end{abstract}

Keywords: Wireless Sensor Networks; Motor Monitoring; Motor Energy Management

\section{Introduction}

Induction motors are the essential driving machines in industry. The motor driven system used in industrial plants consumed about $70 \%$ of all electrical energy consumed by industry [1]-[2]. As an amount of energy loses by the inefficient motors every year, it's important to develop a low-cost in-service motor monitoring and energy management system.

The energy evaluation system in industrial plants is usually implemented with wired communication networks so far. Because of the high cost of installation and maintenance of these cables, it is desired to look for a low-cost, robust, and reliable communication network.

The wireless sensor networks (WSN) is a self-organized network of small sensor nodes with communication and calculation abilities [3]. As an open architecture, self-configuring, robust, and low cost network, it is suitable to meet the requirement

Nathan Ota and Paul[4] discussed the application trends in wireless sensor networks for manufacturing. WSNs can make an impact on many aspects of predictive maintenance (PdM) and condition-based monitoring. WSNs enable automation of manual data collection. PdM applications of WSNs enable increased frequency of sampling. Condition-based monitoring applications benefit from more sensing points and thus a higher degree of automation.

James et al.[5] discussed the robust, self-configuring wireless sensors networks for energy management and concluded that WSN can enable energy savings, diagnostics, prognostics, and waste reduction and improve the uptime of the entire plant.

Bin Lu et al.[6] applied wireless sensor networks in industrial plant energy management systems. A simplified prototype WSN system was developed using the prototype WSN sensors devices, which were composed of a sensor unit, an A/D conversion unit, and a radio unit. However, because the IEEE 802.15.4 standard is designed to provide relaxed data throughput, it is not acceptable in some real-time cases for the large amount of raw data to be transmitted from the motor control center (MCC) 
to the central supervisory station (CSS) .

In this paper, wireless sensor devices are developed and applied in an in-service motor monitoring and energy management system. A DSP is used for data processing at the MCC, and only the processing results are transmitted to the CSS. This approach can greatly reduce the transmission time.

This work is an extension to the previously published work. More details about the communication protocol of the WSN nodes designed in this paper are discussed. A three-level system architecture for in-service motor monitoring and energy management is proposed.

\section{Wireless Sensor Network and IEEE 802.15.4 Standard}

The WSN is a self-organized network with dynamic topology structure, which is broadly applied in the areas of military, environment monitoring, medical treatment, space exploration, business, and household automation [3].

The IEEE802.15.4 standard is a physical layer and MAC sub-layer protocol for WSN, which supports three frequency bands with 27 channels as shown in Figure 1. The $2.4 \mathrm{GHz}$ band defines 16 channels with a data rate of $250 \mathrm{KBps}$. It is available worldwide to provide communication with large data throughput, short delay, and short working cycle. The $915 \mathrm{MHz}$ band in North America defines 10 channels with a data rate of $40 \mathrm{Kbps}$. The $868 \mathrm{MHz}$ band in Europe defines only 1 channel with a data rate of $20 \mathrm{Kbps}$. They provide communication with small data throughput, high sensitivity, and large scales.

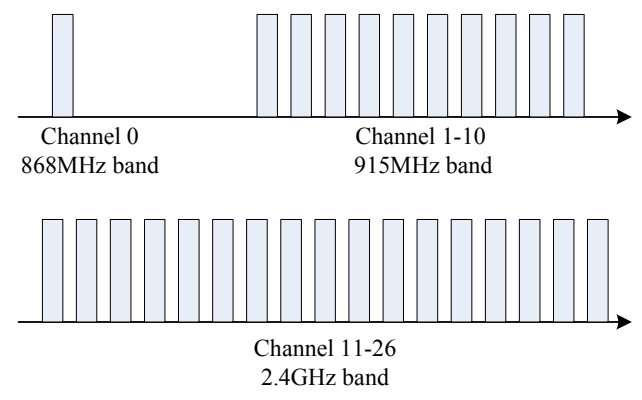

Figure 1. IEEE 802.15.4 frequency bands and channels

The IEEE 802.15.4 supports two network topologies as shown in Figure 2. The star topology is simple and easy to implement. But it can only cover a small area. The peer-to-peer topology, on the other hand, can cover a large area with multiple links between nodes. But it is difficult to implement because of its network complexity.
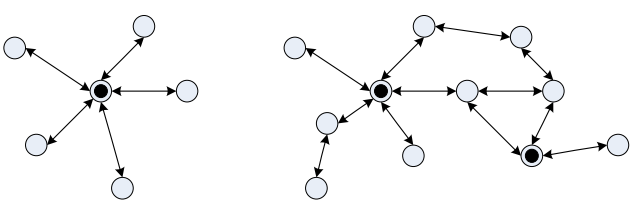

Figure 2. Star (L) and peer-to-peer (R) topologies

An IEEE packet, called physical layer protocol data unit (PPDU), consists of a five-byte synchronization header (SHR) which contains a preamble and a start of packet delimiter, a one-byte physical header (PHR) which contains a packer length, and a payload field, or physical layer service data unit (PSDU), which length varies from 2 to 127 bytes depending on the application demand, as shown in Figure 3.

\begin{tabular}{|c|c|c|c|}
\hline Preamble & $\begin{array}{c}\text { Start of } \\
\text { packet } \\
\text { delimiter }\end{array}$ & $\begin{array}{c}\text { PSDU } \\
\text { Length }\end{array}$ & PHY layer payload \\
\hline 4 bytes $\rightarrow-1$ byte $\rightarrow-1$ byte $\rightarrow$ & $2-127$ bytes $\longrightarrow$ PSDU \\
\hline
\end{tabular}

Figure 3 IEEE 802.15.4 packet structure

\section{Design and Implementation of WSN devices}

\subsection{The front-end devices}

The front-end device is developed with the digital signal processing (DSP) techniques. It is divided into three parts: sensing, signal processing and transmitting unit, as shown in Figure 4.

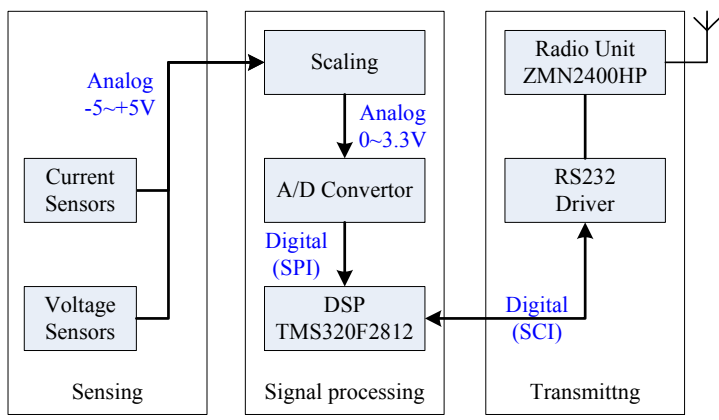

Figure 4 The design of the front-end device

The three parts of the front-end devices are designed and implemented separately on individual PCB's. When constructing the front-end devices, the signal processing unit and the transmitting unit are mounted on the sensing unit and linked by cables with each other, as shown in Figure 5. The flexible design could meet the requirement for different sensors while different motors are monitored. Moreover the sensing unit could be omitted in the case that the current and voltage sensors are already 
equipped in the MCC's in industrial plants. In that case, the transmitting unit can be mounted on the signal processing unit.

The sensing unit consists of two current sensors and two voltage sensors. Both of them are highly accurate Hall effect ones. In the prototype devices used in the laboratory, the current sensor is HNC025A with 0-36 amps RMS current range, \pm $0.6 \%$ accuracy, and $<0.2 \%$ linearity, and the voltage sensor is HNV025A with $100-2500 \mathrm{~V}$ volts RMS current range, $\pm 0.6 \%$ accuracy, and $<0.2 \%$ linearity.
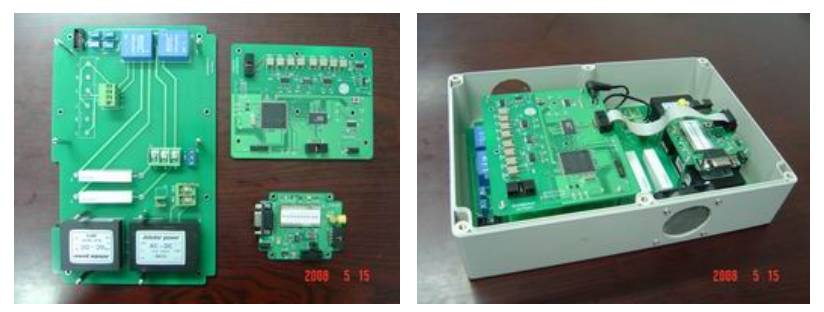

Figure 5 Implementation of the sensing, processing and transmitting unit (WSN node)

The signal processing unit contains three main subunits. The $-5 \mathrm{v}-+5 \mathrm{v}$ analogue voltage signals coming from the sensing unit are firstly scaled into analogue signals in the range of $0-3.3$ volts to meet the requirement of the ADC chip. Then a 12-bit 8-channel ADC is used to sample the analogue waveforms at a certain frequency which can be configured as 2, 4 or $8 \mathrm{KHz}$ in the prototype devices, and convert them into digital signals.

The kernel of the signal processing unit is a 32-bit fixed-point DSP chip TMS320F2812, which has $128 \mathrm{~KB}$ flash memory, $18 \mathrm{~KB}$ internal SRAM. It controls the signal processing and spectrum estimation programs running in a $\mu \mathrm{cOS} / \mathrm{II}$ system.

\subsection{The WSN nodes}

The transmitting unit is implemented with a Cirronet ZMN2400HP wireless module to transform motor running parameters from the front-end device to the CSS. The ZMN2400HP consists of an 8-bit Atmel Mega128 microcontroller, which has $128 \mathrm{~KB}$ flash memory, $4 \mathrm{~KB}$ EEPROM and $4 \mathrm{~KB}$ internal SRAM, and a Chipcon CC2420 radio chip, which is compatible with the IEEE 802.15.4 standard and works at $2.4 \mathrm{GHz}$ band. A more detailed structure of the radio unit is shown in Figure 6.

Generally there are three kinds of nodes in a wireless sensor network: transmitter nodes, which have both sensing and wireless communicating capabilities, the receiver nodes, which have both wireless and wire communicating capability, and relay nodes which have only the wireless communicating capability to relay the data packets in the case that the distances between the transmitter and receiver nodes are beyond the communication range.

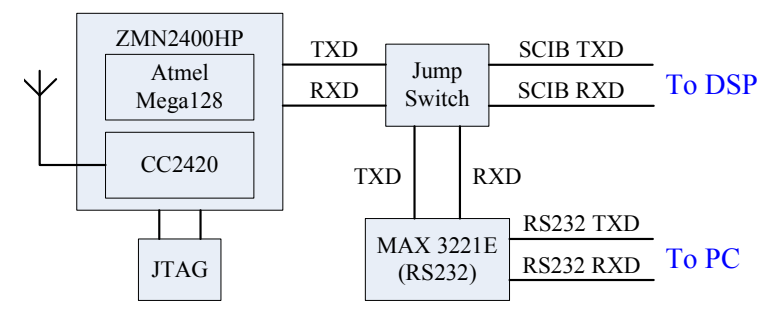

Figure 6 Design of WSN nodes

In the in-services motor monitoring system, most of the WSN nodes are transmitter ones used in the MCC to transmit the processing results to the CSS. As a few receiver and relay nodes are used in the system, all of the three kinds of nodes are implemented based on the same hardware structure to simplify the design. Those full-capability nodes can be configured to act as transmitter, receiver or relay nodes. This gives the reason why the transmitting unit is separated from the signal processing unit in the design of the front-end devices.

Power consumption is the dominating factor in the design of WSN nodes. However in this specific application, the power consumption is no longer a problem to be considered because the WSN nodes are installed at such locations as a MCC or a CSS, where the power supply is available. So the WSN nodes are designed to be powered by $\mathrm{AC} / \mathrm{DC}$ converters.

Additionally, when a WSN node works together with a processing unit, it can also be powered by the same power module in the processing unit.

\subsection{The communication protocol}

Generally the data transmissions are initiated by the front-end devices. When the signal processing unit gets the results ready, it makes an interrupt request to the transmitting unit, which acknowledges the request and receives the data through the asynchronous serial ports and then transmits them to the CSS.

There are two kinds of data transmissions which are initiated by the CSS. The first one is the raw data transmission. When more detailed analysis needs to be made, the raw currents data must be sent to the CSS, where the raw data are processed and analyzed by the more powerful PC. When this situation occurs, a raw data request is sent by the CSS to the front-end device, which then gathers some raw data 
and divides them into several data block packets to send to the CSS one by one. Each packet contains a 2-byte data block number and a 64-byte data block. The front-end device waits for an acknowledge packet with the 2-bye data block number sent back by the CCS before continuing to send the next one. When the CSS receives a data block packet with a flag to identify it as the last one, it sends back a raw data ending acknowledge packet and the raw data transmission ends. See Figure 7.

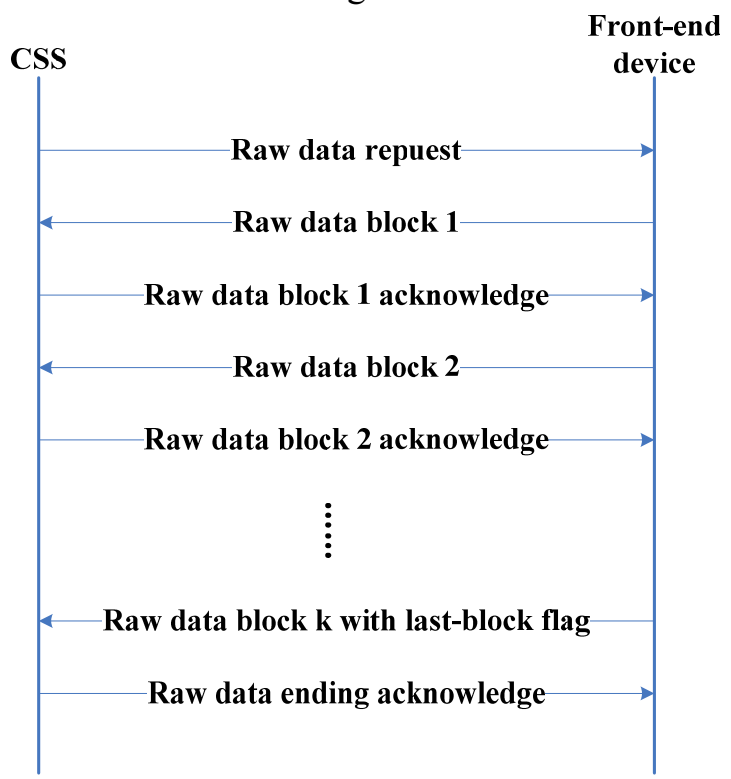

Figure 7 Raw data transmission

Some abnormal cases are handled simply during the raw data transmission. If the front-end device can't receive an expected acknowledge packet in a given period of time, it simply ends the raw data transmission. If the CSS can't receive an expected raw data block packet in a given period of time, it resends the acknowledge packet 3 times before it ends the raw data transmission. Obviously, the timeout set for the front-end devices is at least 3 times larger than the timeout set for the CSS.

The second data transmission initiated by the CSS is the configuration. A configuration packet is sent to the front-end devices which guided them to configure the processing parameters, such as the motor poles, motor slots, current and/or voltage sensors errors, etc.

Additionally, some log data are transmitted, including the conditions of the nodes, repeaters (routers), and coordinators. When the network fails, the $\log$ data are stored in the EEPROM temporarily and sent to the CSS as soon as the connection is rebuilt.

There are nine kinds of communication packets, as illustrated in table 1 .
Table 1 Communication packet types

\begin{tabular}{|c|c|c|}
\hline Type & Description & Direction \\
\hline 0x00 & Processing results request & CSS $\rightarrow$ FED \\
\hline 0x11 & Raw data request & CSS $\rightarrow$ FED \\
\hline 0x12 & Configuration & CSS $\rightarrow$ FED \\
\hline 0x13 & Raw data block acknowledge & CSS $\rightarrow$ FED \\
\hline 0x14 & Raw data ending acknowledge & $\mathrm{CSS} \rightarrow$ FED \\
\hline 0x21 & Processing results data & FED $\rightarrow$ CSS \\
\hline 0x22 & Raw data block & FED $\rightarrow$ CSS \\
\hline 0x23 & Configuration acknowledge & FED $\rightarrow$ CSS \\
\hline 0x2A & Log data & Nodes $\rightarrow$ CSS \\
\hline
\end{tabular}

Note: FED stands for "front-end devices".

\subsection{ID table}

A coordinator at CSS manages all the nodes in the network by an ID table. A node registers to the coordinator by reporting its ID after it powers on or resets. The coordinator communicates with each node in the ID table in turn to get the processing results from the front-end devices. In this way, the communicating conflict can be avoided. If the coordinator couldn't receive any data from a node in a given time, it deletes its ID from the table.

The ID table is defined as follows:

typedef struct

\{

// node ID

USIGN8 ucNodeID;

// node address

USIGN16 uNodeShortAddr;

// request fail counter

USIGN8 ucReqFailCounter;

\}NODE_ID;

typedef struct

\{

// node counter

USIGN8 nodeNum;

NODE_ID nodeId[MAX_NODE_NUM];

\}NODE_ID_TABLE;

Table 2 ID table updating

\begin{tabular}{|c|c|c|c|}
\hline C1 & C2 & C3 & Update \\
\hline N & N & - & Add new node ID \\
\hline N & Y & - & Set the node ID in the record \\
\hline Y & N & - & Set the node address in the record \\
\hline Y & Y & Y & Set new node address in the record \\
\hline Y & Y & N & No action \\
\hline
\end{tabular}


The ID table is updated according to the combination of three conditions as described in table 2. Here condition 1 (C1) is that the node ID is in the table. Condition 2 (C2) is that the node address is in the table. Condition 3 (C3) is that the node address changed.

\subsection{The abnormal handling}

A WSN node resets its main CPU and the CC2420 chip and searches for the network again in three cases. First, it can't connect to the network in a given period of time after it powered on. Second, it can't receive the acknowledgement when it tries to register its ID to the coordinator at CSS after connecting to the network. Last, it doesn't receive the processing results request in a given period of time during a connecting session.

A repeater (router) transmits data between nodes and the coordinator. It's more complex to judge a repeater's condition because both the nodes and the coordinator could reset in some cases. Some actions are made according to the combination of five conditions as described in table 3 . Here condition $1(\mathrm{C} 1)$ is that the repeater has received data from the coordinator. Condition 2 (C2) is that the repeater has received data from nodes. Condition 3 (C3) is that the repeater has got an overtime during transmitting data with the coordinator. Condition 4 (C4) is that the repeater has got an overtime during transmitting data with nodes. Condition 5 (C5) is that the repeater has got an overtime during registering to the network.

The coordinator handles abnormal situations in two cases. It resets its main CPU and CC2420 chip to rebuild the network if no nodes register to it in a given period of time when network initiating or all IDs are deleted from its records.

Table 3 Repeater abnormal processing

\begin{tabular}{|c|c|c|c|c|c|}
\hline C1 & $\mathrm{C} 2$ & $\mathrm{C3}$ & $\mathrm{C4}$ & C5 & Action \\
\hline \multirow{2}{*}{$\mathrm{N}$} & \multirow{2}{*}{$\mathrm{N}$} & \multirow{2}{*}{-} & \multirow{2}{*}{ - } & $\mathrm{N}$ & Wait for data \\
\hline & & & & $\mathrm{Y}$ & Reset \\
\hline \multirow{2}{*}{$\mathrm{N}$} & \multirow{2}{*}{$\mathrm{Y}$} & \multirow{2}{*}{-} & $\mathrm{N}$ & \multirow{2}{*}{ - } & Wait for data \\
\hline & & & $\mathrm{Y}$ & & Reset \\
\hline \multirow{2}{*}{$\mathrm{Y}$} & \multirow{2}{*}{$\mathrm{N}$} & $\mathrm{N}$ & \multirow{2}{*}{ - } & \multirow{2}{*}{ - } & Wait for data \\
\hline & & $\mathrm{Y}$ & & & Reset \\
\hline \multirow{4}{*}{ Y } & \multirow{4}{*}{$\mathrm{Y}$} & $\mathrm{N}$ & $\mathrm{N}$ & \multirow{4}{*}{ - } & No \\
\hline & & $\mathrm{N}$ & $\mathrm{Y}$ & & \multirow{3}{*}{ Reset } \\
\hline & & $\mathrm{Y}$ & $\mathrm{N}$ & & \\
\hline & & $\mathrm{Y}$ & $\mathrm{Y}$ & & \\
\hline
\end{tabular}

\section{In-Service Motor Energy Management}

\subsection{Motor Energy Management Architecture}

It's a complicated system engineering to apply motor energy management. First of all, a motor asset database should be established to record the basic information of all the in-service motors. Then the necessary on-line monitoring should be made on the motor systems to acquire the energy consumption, efficiency, power factor, load rate, etc., and motor monitoring database should be made to record all them. After that, the motor energy management can be made by applying the signal processing, condition monitoring and diagnosis, health management, and energy consumption and saving analysis technologies.

The in-services motor monitoring system can be divided into three levels: the motor condition collection at the base level, data processing and condition monitoring in the middle, and the energy consumption and saving analysis at the top. In this paper, such a three levels system architecture is proposed, which composed of a data acquisition platform, a communication platform, a condition monitoring platform, a motor energy management platform, and an energy consumption and saving analysis platform, as shown in Figure 8. An in-service motor efficiency monitoring and energy management system was developed based on this architecture.

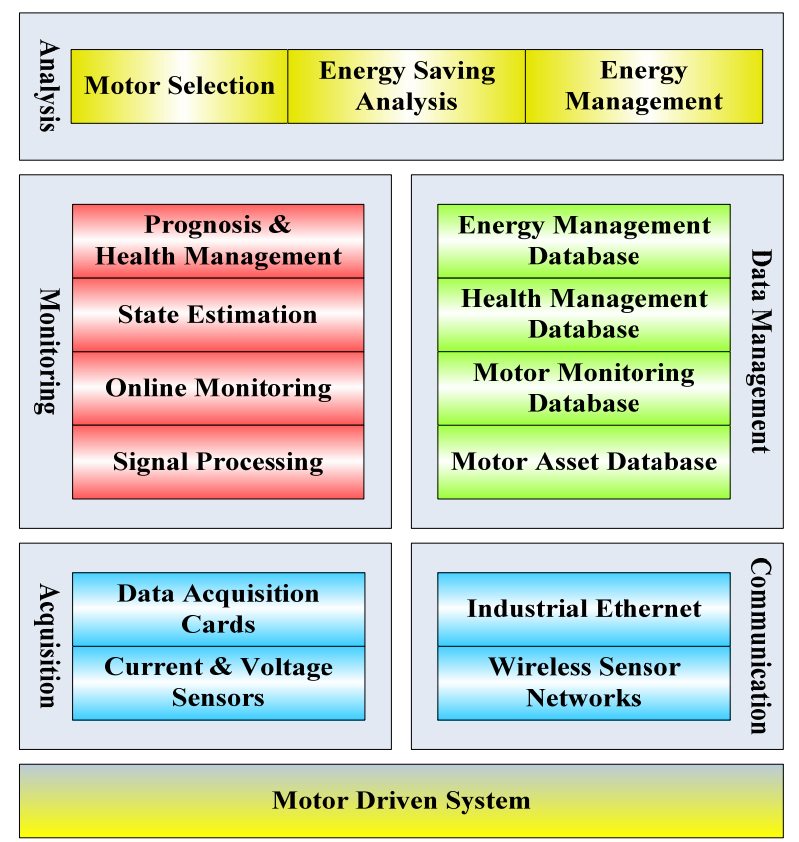

Figure 8 The three-level system architecture 


\subsection{Motor Efficiency Estimation}

The energy usage evaluation is one of the basic functions of an in-service motor energy management system. The most important among the energy evaluation functions is the estimation of motor efficiency, which is defined as the ratio of the motor shaft output power PO to the input power PI as (1), and the difference between them is the power losses which are classified as stator copper loss WS, rotor copper loss WR, core loss WC, friction and windage loss WFW, and stray load loss WLL, as given by (2)[7].

$$
\begin{aligned}
& \eta=\frac{P_{O}}{P_{I}} \times 100 \% \\
& W_{L}=P_{I}-P_{O}=W_{S}+W_{R}+W_{C}+W_{F W}+W_{L L}
\end{aligned}
$$

There are many methods to determine the motor efficiency. Generally they are divided into three groups, direct detection methods, indirect detection methods (also known as segregated loss methods), and inference methods [7]-[10].

Many direct and indirect methods have been adopted by some international standards such as IEEE 112-B, IEC 34-2, and JEC 37. The Chinese national standard for motor efficiency determination is GB1032-2005. The methods defined in the standards are agreement. The main difference of them is how to determine the stray load loss.

The inference methods estimate the motor efficiency by calculations after some simple experiments. The standard slip method presumed that the percentage of the load is proportional to the ratio of the measured slip to the full-load slip. Improvements are made by correcting the rated nameplate speed for voltage variations as given by (4)[7].

$$
\begin{aligned}
& \eta=\frac{\text { slip }}{\operatorname{slip}_{\text {rated }}} \cdot \frac{P_{O, \text { rated }}}{P_{I}} \\
& \eta=\frac{\operatorname{slip}}{\operatorname{slip}_{\text {rated }}} \cdot \frac{P_{O \text {,rated }}}{P_{I}} \cdot\left(\frac{V}{V_{\text {rated }}}\right)^{2}
\end{aligned}
$$

The current methods assume that the percentage of load is proportional to the ratio of the measured current to full-load current. An improved current method may give a more accurate efficiency estimate as given by (6)[7].

$$
\begin{aligned}
& \eta=\frac{I}{I_{\text {rated }}} \cdot \frac{P_{O, \text { rated }}}{P_{I}} \\
& \eta=\frac{2 I-I_{\text {noload }}}{2 I_{\text {rated }}-I_{\text {noload }}} \cdot \frac{P_{O \text {,rated }}}{P_{I}}
\end{aligned}
$$

The third type of reference methods is torque methods. The motor efficiency is defined in (7) in terms of the shaft torque and the rotor speed.

$$
\eta=\frac{T_{\text {shaft }} \cdot \omega_{r}}{P_{I}}
$$

The methods described above are bench testing which requires the motor to be tested in a laboratory environment that may be different from the original working site. Another disadvantage is that they require the motor to be removed from service. They cannot be directly used for the in-service motors.

The motor current signature analysis (MCSA) method is a non-intrusive in-service testing method, which only rely on terminal voltages and currents while a motor is running. The motor is tested in situ, that means motor's original working condition is maintained. Without the installation of sensors, such as speed, torque, and temperature ones, on the motor body, it is easy to monitor the in-service motors in industrial plants.

In this paper, the MCSA method is used to estimate motor efficiency from the motor stator current and voltage signals collected at the power supply. The efficiency is calculated using (8) where the air gap torque TAG is obtained using (9) [11] and the rotor speed $\omega r$ is estimated using rotor slot harmonics and spectral estimation techniques using (10) [12].

$$
\begin{aligned}
& \eta=\frac{P_{M}}{P_{E}}=\frac{T_{A G} \cdot \omega_{r}-\left(L_{C O R E}+L_{F W}+L_{S}\right)}{P_{E}} \\
& T_{A G}=\frac{\text { Poles }}{2 \sqrt{3}}\left\{\begin{array}{c}
\left(i_{A}-i_{B}\right) \cdot \int\left[u_{C A}-R\left(i_{C}-i_{A}\right)\right] d t \\
-\left(i_{C}-i_{A}\right) \cdot \int\left[u_{A B}-R\left(i_{A}-i_{B}\right)\right] d t
\end{array}\right\} \\
& \omega_{r}=\frac{2 \pi}{z_{r}}\left(f_{s h} \pm f_{1}\right)
\end{aligned}
$$

The losses are simplified by assuming the combined iron core, friction, windage and stray load losses to be $5 \%$ of rated input power according to IEEE Std-112 and [13].

\subsection{In-Service Motor Monitoring}

In order to evaluate the energy usage, 8 motor condition parameters are estimated and/or calculated, including the current root mean square (Irms), the voltage root mean square (Urms), the input power $(\mathrm{PE})$, the power factor $(\cos \varphi)$, the rotor speed $(\omega$ r), the shaft torque (TAG), the output power (PM), and the efficiency $(\eta)$, as shown in Figure 9. 


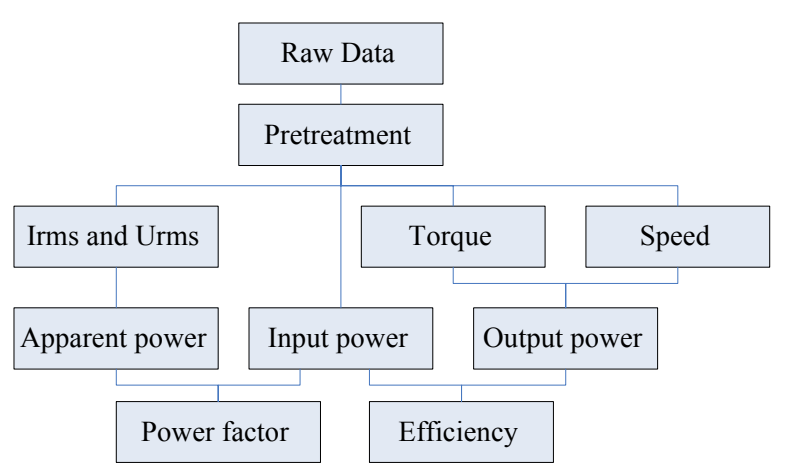

Figure 9 Motor condition parameters calculation

Besides the rotor speed and the shaft torque, other 5 parameters can be obtained by (11)-(15).

$$
\begin{aligned}
& I_{r m s}=\sqrt{\frac{1}{N} \sum_{m=1}^{N} i_{m}^{2}} \\
& U_{r m s}=\sqrt{\frac{1}{N} \sum_{m=1}^{N} u_{m}^{2}} \\
& P_{E}=\frac{1}{N} \sum_{m=1}^{N} u_{m} i_{m} \\
& \cos \varphi=\frac{P}{S}=\frac{P}{\sqrt{3} \cdot U \cdot I} \\
& P_{M}=\frac{2 \pi n}{60} T
\end{aligned}
$$

\section{The implementation of a prototype system}

\subsection{The application of WSN}

The in-service motor monitoring and energy management system is constructed based on the DSP devices and WSN nodes presented above.

Based on the MCSA technology, the in-service motor monitoring and energy management system consists of some front-end devices, which are installed at the MCC to acquire and analyze the motors current and voltage signals, and a back-end CSS, which gathers and stores the analysis results from the front-end devices and estimates the motor conditions. The communication between them is based on WSN architecture. The system architecture is illustrated in Figure 10.
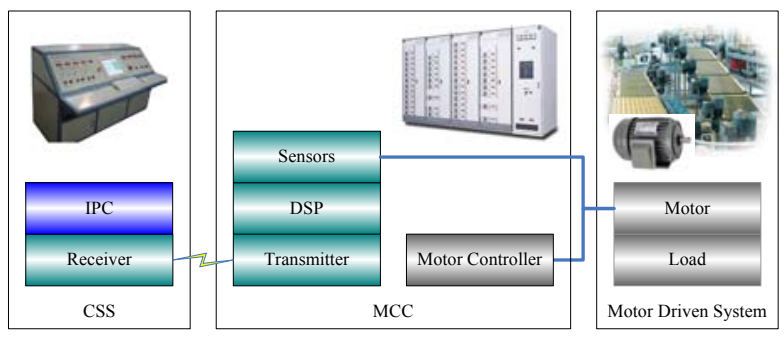

Figure 10 In-service motor monitoring and energy management system
At MCC, the motor stator line current and voltage signals are acquired by the sensing unit and analyzed by the signal processing unit to estimated/calculated all the 8 parameters mentioned in section 4.3. At last, the results are transmitted to the CCS by the transmitting unit over the wireless sensor networks.

At CCS, all the 8 parameters are stored in the database and displayed with instantaneous values and wave charts as shown in Figure 11.
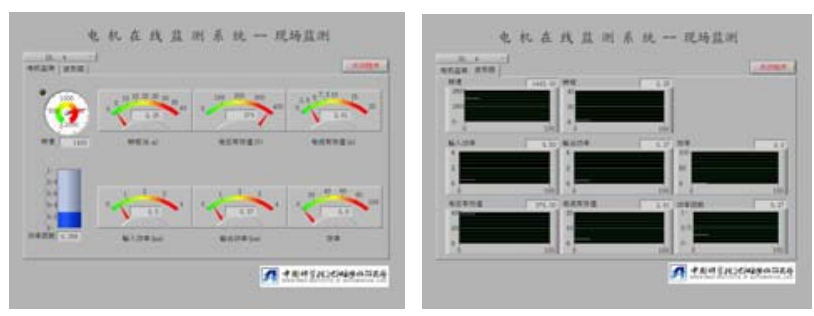

Figure 11 Motor condition monitoring

\subsection{Data throughput}

As described in section 2, the PSDU length can vary from 2 to 127 bytes in a IEEE 802.15.4 data packet. In the proposed system, the PSDU is totally 32 bytes long with 1-byte motor ID, 1-byte frame type, 2-byte counting number, 4-byte voltage, 4-byte current, 4-byte speed, 4-byte torque, 4-byte input power 4-byte output power, 2-byte efficiency, and 2-byte power factor. Apparently, one result can be transmitted in one data packet.

To meet the requirement of signal processing, 4 channels of current and voltage signals are sampled synchronously at $4 \mathrm{KHz}$ frequency for 1 second to get 50 cycles of $50 \mathrm{~Hz}$ waveforms. Another 2 seconds are spent on calculating and transmitting the results. So every 3 seconds, a data packet is sent to the CSS from one front-end device.

That transmitting time and data throughput requirement is enough to be implemented in an IEEE 802.15.4 WSN with the standard latency 6-60 $\mathrm{ms}$ and data throughput 250KBps.

To check the maximum communication abilities between the WSN nodes, a simple test is made in which real size data packets are continuously send from a transmitter to a receiver in $300 \mathrm{~ms}$ with each packet sent within an specified interval (Is). The packets sent from the transmitter (Ps) and the packets received by the receiver $(\mathrm{Pr})$ are counted. Then the real receiving interval (Ir), average packets received per second $(\mathrm{Pa})$, and the packets lost rate $(\mathrm{Lr})$ are calculated. The test results are illustrated in Table 4.

From the test results, it can be seen that the minimum packets receiving interval is about 0.015 
seconds. In other words, maximum 66.7 packets can be received every second on average. If the transmitter sends packets faster than that, the communication becomes worse with packets lost rate getting higher.

Table 4 Communication abilities test

\begin{tabular}{|c|c|c|c|c|c|}
\hline Is & Ps & Pr & Ir & Pa & Lr \\
\hline 0.100 & 2976 & 2976 & 0.010 & 9.92 & 0.0000 \\
\hline 0.050 & 5887 & 5887 & 0.005 & 19.6 & 0.0000 \\
\hline 0.030 & 9691 & 9691 & 0.003 & 32.3 & 0.0000 \\
\hline 0.025 & 11567 & 11567 & 0.002 & 38.5 & 0.0000 \\
\hline 0.020 & 14310 & 14310 & 0.002 & 47.7 & 0.0000 \\
\hline 0.015 & 18791 & 18790 & 0.001 & 62.6 & 0.0053 \\
\hline 0.010 & 22577 & 19537 & 0.001 & 65.1 & 13.4650 \\
\hline 0.005 & 29718 & 18851 & 0.001 & 62.8 & 36.5671 \\
\hline
\end{tabular}

\subsection{Laboratory Test and Plant Application}

A laboratory test is made in a prototype system including an $\mathrm{MCC}$, a CSS, and four Y100L2-4

induction motors (4-pole, $3 \mathrm{KW}$, $380 \mathrm{~V}, 6.8 \mathrm{~A})$ with four $4 \mathrm{KW} \quad \mathrm{DC}$

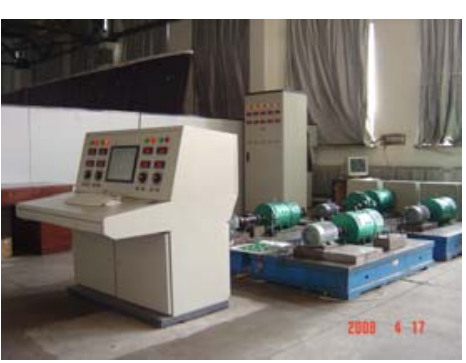

Figure 11 Test system generators as their loads, as shown in Figure 11.

In the CCS, a WSN receiver node is used as a coordinator of the network. Four front-end devices are installed in the MCC to acquire the current and voltage signals of the four test motors. When started, they search and connect to the coordinator automatically to setup a star wireless network. Then the coordinator sends a query packet to one of the 4 front-end nodes every second and receives a data packet sent back on the request. In this way, the motor monitoring results are successfully transmitted to the CSS constantly.

The motors are tested from no load to full load with intervals of $12.5 \%$ load. Signals are sampled and analyzed for 120 seconds at each load point. That means totally 4 (motors) * 9 (load point per motor) * 120 (seconds per load point) / 3 (seconds for one packet $)=1440$ packets are transmitted from 4 front-end devices to the CCS. As only one packet is sent to the coordinator from one of the 4 front-end monitoring devices every second, the data throughput is enough to transmit the data packets, and there is no packet lost in the laboratory test.
The system had been applied in a plant to monitor four pumping motors and three heating ovens as illustrated in Figure 12..
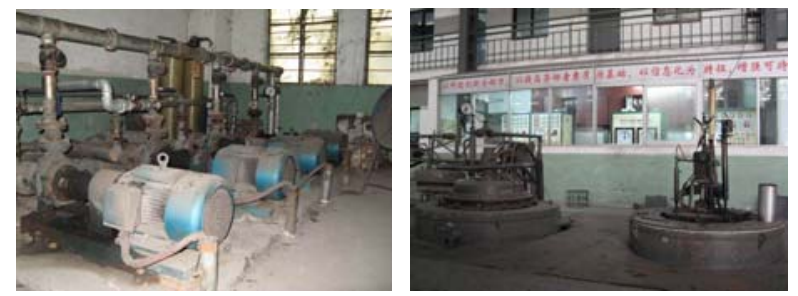

Figure 12 Pumping Motors and Heating Ovens

\section{Conclusion}

This paper presents the development of wireless sensor devices in the in-service motor monitoring and energy management system which is used to evaluate the condition of motors used in industrial plants.

The front-end device consists of a sensing unit to acquire the motor current and voltage signals, a DSP unit to perform the motor current signature analysis, and a radio unit to transmit the results to a central supervisory station over the wireless networks based on the IEEE 802.15.4 standard. As the analysis and calculation are made at the front end and only the results are transmitted by the wireless network, this approach greatly reduce the transmission time. That makes the proposed system acceptable in real-time cases.

\section{Acknowledgment}

This work was supported by the National Hi-Tech R\&D Program of China (863 Program) under Grant No. 2007AA041201, and the Innovative Project of the Advanced Manufacturing Base of Chinese Academy of Sciences under Grant No. CX07-03-003.

\section{References}

[1] U.S. Department of Energy, Sensors \& Automation 2007 Annual Portfolio Review, Rosemont, Illinois, June 2007.

[2] Electrical Industrial Association of China, "The New Trend of Motor Energy-saving", Electrical Industry, April 2006, pp. 50-52

[3] Yu Haibin, Zeng Peng, and Liang Wei, Intelligent Wireless Sensor Networks, 1st ed., Science Press: Beijing, 2006, pp. 11.

[4] Nathan Ota* and Paul Wright, "Trends in wireless sensor networks for Manufacturing", Int. J. Manufacturing Research, Vol. 1, No. 1, 2006, pp. 3-17 
[5] James E. Hardy, Wayne W. Manges, Jose A. Guitierrez and Phani Teja V. Kuruganti, "Wireless Sensors and Networks for Advanced Energy Management", 2005 ACEEE Summer Study on Energy Efficiency in Industry, July 19th - 22nd, 2005, New York

[6] Bin LU, Thomas G. Habetler, Ronald G. Harley, and Jose A. Gutierrez, “Applying Wireless Sensor Networks in Industrial Plant Energy Management Systems - Part I \& Part II", 5th IEEE Conference on Sensors, Oct. 2005, pp.145-150

[7] B. Lu, T. G.. Habetler, and R. G.. Harley, "A Survey of Efficiency-Estimation Methods for In-Servce Induction Motors", IEEE Trans. Industry Applications, vol. 42, no. 4, pp.924-933, Jul./Aug. 2006.

[8] J. Hsu, J. Kueck, M. Olszewski, D. Casada, P. Otaduy, and L. Tolbert, "Comparison of induction motor field efficiency estimation methods," IEEE Trans. Ind. Appl., vol. 34, no. 1, 1998.

[9] A. Wallance, A. Von Jouanne, E. Wiedenbrug, E. Matheson, and J.Douglass, "A laboratory assessment of in-service and non-intrusivemotor efficiency testing methods," Electric Power Components andSystems, vol. 29, pp. 517-529, 2001.

[10] J. D. Kueck, "Development of a method for estimating motor efficiency and analyzing motor condition," IEEE Pulp and PaperIndustry Technical onference, pp. 67-72, June 1998.

[11] J. Hsu and B.P. Scoggins, "Field Test of Motor Efficiency and Load Changes through Air-gap Torque", IEEE Trans. Energy Convers., Vol.10, No.3, 1995(11), pp.477-483

[12]X.Z, Che, J.T. Hu, and Q.J. Guo, “An Slot Harmonics Detection-Based Approach to Speed Estimation in a Sensorless Induction Motor", Chinese Journal of Scientific Instrument, Vol.29, No.4S, 2008(4), pp. 414-417

[13] "In-plant Electric Motor Loading and Efficiency Techniques", Ontario Hydro, Toronto, ON, Canada, Market Literature 